Sharif University of Technology
Scientia Iranica
Transactions E: Industrial Engineering
hCIENTIA

\title{
On accuracy function and distance measures of interval-valued Pythagorean fuzzy sets with application to decision making
}

\author{
T. Kumar ${ }^{\mathrm{a}}$, R.K. Bajaj ${ }^{\mathrm{b}, *}$, and M. Dilshad Ansaric \\ a. SRM Institute of Science and Technology, Delhi-NCR Campus, Ghaziabad (UP), India. \\ b. Jaypee University of Information Technology, Waknaghat, Solan (HP), India. \\ c. CMR College of Engineering \& Technology, Kandlakoya, Hyderabad (TS), India.
}

Received 16 August 2018; received in revised form 10 October 2018; accepted 26 January 2019
KEYWORDS
Interval-valued
Pythagorean fuzzy
numbers;
Score function;
Accuracy function;
Distance measures;
Weighted averaging
operators.

\begin{abstract}
In the present paper, a new accuracy function is provided to overcome the limitations of the existing score/accuracy functions for interval-valued Pythagorean Fuzzy Sets (PFSs). The proposed accuracy function is validated and discussed in detail through illustrative examples. Furthermore, a new distance measure for interval-valued Pythagorean fuzzy numbers is proposed and used in terms of the existing weighted averaging operators. Finally, with regard to the proposed accuracy function, distance measure, and weighted averaging operators, a numerical example of Multi-Criteria Decision Making (MCDM) process is presented to validate the methodology.
\end{abstract}

(C) 2020 Sharif University of Technology. All rights reserved.

\section{Introduction}

Fuzzy sets [1] have been used to express imprecise or vague information in various fields of real-world application. Intuitionistic Fuzzy Set (IFS) proposed by Atanassov [2] has been found a highly adjustable framework to grapple with uncertainty with a certain amount of hesitation arising from imperfect or vague information. The concept of IFS has been widely studied and applied to deal with uncertainties and hesitancy inherent in practical circumstances. The most significant characteristic of an IFS is that it assigns a number from the unit interval $[0,1]$ to

\footnotetext{
*. Corresponding author.

E-mail addresses: tanujkumar.o@ncr.srmuniv.ac.in (T.

Kumar); rakesh.bajaj@juit.ac.in (R.K.Bajaj);

m.dilshadcse@gmail.com (M. Dilshad Ansari)
}

every element in the domain of discourse, a degree of membership, and a degree of non-membership along with the degree of indeterminacy, whose total sum equals unity. In the literature, IFSs and interval-valued IFSs comprehensively span applications to the fields of decision making problems [3], pattern recognition, sales analysis financial services, medical diagnosis, etc.

Pythagorean Fuzzy Set (PFS) [4] is an efficient generalization of IFS characterized by the inequality that the squared sum of membership and nonmembership values is less than or equal to one. Yager and Abbasov [5] stated that in some practical MultipleCriteria Decision Making (MCDM) problems, it is viable that the sum of the degree of the membership and the degree of non-membership in a particular alternative provided by a decision maker may be greater than one, where it would not be feasible to use IFS. PFS has proven more proficient than IFS in representing and handling vagueness, impreciseness, and uncertainties in various decision making processes. Since the span of 
membership degree of PFS is greater than that of IFS, it may be stated that it is more generalized and has wider applicability.

Yager [4] also developed some aggregation operations under the PFS environment. Furthermore, Yager and Abbasov [5] investigated the correspondence between the degrees of Pythagorean membership and complex numbers and found that Pythagorean membership degrees were only subsets of the complex numbers, called $\Pi-i$ numbers. Utilizing PFSs for solving MCDM problems, Zhang and Xu [6] enhanced the existing technique for order of preference by similarity to ideal solution. PFSs were generalized to intervalvalued PFSs by Peng and Yang [7], who provided some interval-valued Pythagorean fuzzy aggregation operators for handling the related problems. Various new operators with different properties and applications to the field of decision making have also been presented by Peng [8]. A ranking method for Pythagorean fuzzy numbers as well as Interval-Valued Pythagorean Fuzzy Numbers (IVPFNs) was proposed by Zhang [9] by taking the idea of closeness index into account.

In the literature, the notion of distance measures plays a key role in the fuzzy set theory and in the application fields such as MCDM problems [10,11], pattern recognition, medical diagnosis, financial services, etc. Recently, various researchers have proposed different types of distance measures for different types of sets, viz. fuzzy sets, IFSs, and PFSs [12]. The Hamming distance, Euclidean distance, and Hausdorff distance measures are some popular and widely utilized distance measures in the application and research world of soft computing [13-19]. Zhang and $\mathrm{Xu}$ [6] provided a new measure for Pythagorean fuzzy numbers and applied it to an MCDM problem. Furthermore, Li and Zeng [20] stated that the strength and direction of commitment would play an important role in well describing PFNs.

They considered four fundamental parameters, namely membership, non-membership, strength, and direction of commitment of PFNs, and provided some distance measures. Most recently, Liu et al. [21] introduced important distance measures for IVPFNs along with their generalized, weighted, and ordered weighted versions. In addition, they proposed some generalized probabilistic distance measures and various important operators and used them for MCDM problems. This study incorporates a new accuracy function for IVPFNs to overcome the limitations of the score/accuracy function in the existing methodologies and employs IVPFNs for solving an MCDM problem.

Different parts of this paper are organized as follows. First, the basic notation for Pythagorean fuzzy numbers is presented along with their corresponding scores and accuracy functions in Section 2. Then, the notion of IVPFNs is discussed and the shortcomings in the existing score/accuracy functions of IVPFNs are investigated in Section 3. Also, in this section, a new accuracy function for IVPFNs will be proposed to handle the stated shortcomings. In Section 4, first, some exiting distance measures and weighted averaging operators are listed for IVPFNs and then, a new interval-valued Pythagorean fuzzy $p$-distance measure for IVPFNs is introduced in order to overcome the shortcomings in the existing methodology. In Section 5, procedural steps of the proposed algorithm are provided for an MCDM problem. Subsequently, a numerical example is solved to validate the algorithm as well as the applicability of the proposed accuracy function, IVPF $p$-distance measure, and the weighted averaging operators. Based on the illustrative example of the MCDM problem, some important remarks on the limitations of the existing methods are given in Section 6. Finally, Section 7 provides the concluding remarks and the scope for future work.

\section{Pythagorean fuzzy numbers and their score and accuracy functions}

Some fundamentals related to PFSs/numbers along with their score/accuracy functions are presented in this section.

A PFS over $U$ (domain) is defined by Yager [4] as:

$$
P=\left\{<u, \mu_{P}(u), \nu_{P}(u)>\mid u \in U\right\},
$$

where $\mu_{P}: U \rightarrow[0,1]$ and $\nu_{P}: U \rightarrow[0,1]$ are the membership and non-membership functions such that $0 \leq\left(\mu_{P}(u)\right)^{2}+\left(\nu_{P}(u)\right)^{2} \leq 1$. The numbers $\mu_{P}(u)$ and $\nu_{P}(u)$ denote the degrees of membership and nonmembership of $u \in U$ in $P$, respectively. For each PFS $P \in U$, the quantity $\pi_{P}(u)=\sqrt{1-\mu_{P}^{2}(u)-\nu_{P}^{2}(u)}$ represents the degree of indeterminacy of $u \in U$.

For simplicity, Zhang and $\mathrm{Xu}$ [6] called the pair $\left(\mu_{P}(u), \nu_{P}(u)\right)$ as a Pythagorean fuzzy number denoted by $p=\left(\mu_{P}, \nu_{P}\right)$. Peng and Yang [7] defined an intervalvalued $\mathrm{PFS}$ in $U$ given by:

$$
\begin{gathered}
P=\left\{u, \mu_{p}(u)=\left[\underline{\mu}_{p}(u), \bar{\mu}_{p}(u)\right],\right. \\
\left.\nu_{p}(u)=\left[\underline{\nu}_{p}(u), \bar{\nu}_{p}(u)\right] \mid u \in U\right\},
\end{gathered}
$$

where $\left[\underline{\mu}_{p}(u), \bar{\mu}_{p}(u)\right]$ and $\left[\underline{\nu}_{p}(u), \bar{\nu}_{p}(u)\right]$ denote the membership and non-membership degrees of $u$ in $P$, respectively, under the condition $0 \leq\left(\bar{\mu}_{p}(u)\right)^{2}+$ $\left(\bar{\nu}_{p}(u)\right)^{2} \leq 1$. Here, $\underline{\mu}_{p}(u)=\inf \mu_{p}(u), \bar{\mu}_{p}(u)=$ $\sup \mu_{p}(u), \underline{\nu}_{p}(u)=\inf \nu_{p}(u), \bar{\nu}_{p}(u)=\sup \nu_{p}(u)$ for all $u \in U$. The degree of indeterminacy $\pi_{p}(u)=$ $\left[\underline{\pi}_{p}(u), \bar{\pi}_{p}(u)\right]$ for all $u \in U$ is called the interval-valued 
Pythagorean fuzzy index of $u$ in $P$, where:

$$
\begin{aligned}
& \underline{\pi}_{p}(u)=\sqrt{1-\left(\bar{\mu}_{p}(u)\right)^{2}-\left(\bar{\nu}_{p}(u)\right)^{2}}, \\
& \bar{\pi}_{p}(u)=\sqrt{1-\left(\underline{\mu}_{p}(u)\right)^{2}-\left(\underline{\nu}_{p}(u)\right)^{2}} .
\end{aligned}
$$

Yager and Abbasov [5] used another representation of PFN as $p=\left(r_{P}, d_{P}\right)$, where $r_{p}$, and $d_{p} \in[0,1]$ are called the strength and direction of the commitment of $p$, respectively. There is a one-to-one correspondence between $\left(\mu_{p}, \nu_{p}\right)$ and $\left(r_{p}, d_{p}\right)$, given by $\mu_{p}=$ $r_{p} \cos \left(\theta_{p}\right), \nu_{p}=r_{p} \sin \left(\theta_{p}\right)$, where $\theta_{p}=\arccos \left(\mu_{p} / r_{p}\right)$ and $d_{p}=1-\frac{2 \theta_{p}}{\pi}$.

Furthermore, Yager and Abbasov [5] showed that the Pythagorean membership degrees were contained in the class of complex numbers, denoted by $\prod-i$. Therefore, they presented PFN $p=\left(\mu_{p}, \nu_{p}\right)$ as $p=$ $r e^{-i \theta}$, where $\mu_{p}=r_{p} \cos (\theta)$ and $\nu_{p}=r_{p} \sin (\theta)$.

In order to compare two PFNs, Yager [4] proposed the following formula:

$$
V(p)=\frac{1}{2}+r_{p}\left(d_{p}-\frac{1}{2}\right)=\frac{1}{2}+r_{p}\left(\frac{1}{2}-\frac{2 \theta_{p}}{\pi}\right) .
$$

Let $p_{1}=\left(r_{p_{1}}, d_{p_{1}}\right)$ and $p_{2}=\left(r_{p_{2}}, d_{p_{2}}\right)$ be two PFNs, then:

- If $V\left(p_{1}\right)>V\left(p_{2}\right)$, then $p_{1} \succ p_{2}$;

- If $V\left(p_{1}\right)=V\left(p_{2}\right)$, then $p_{1} \sim p_{2}$.

Furthermore, for comparing two Pythagorean fuzzy numbers, Zhang and $\mathrm{Xu}$ [6] proposed a score function of $p=\left(\mu_{p}, \nu_{p}\right)$ given as:

$$
s(p)=\left(\mu_{p}\right)^{2}-\left(\nu_{p}\right)^{2},
$$

where $s(p) \in[-1,1]$.

Based on the score function (Eq. (2)), Zhang and $\mathrm{Xu}$ [6] gave the comparison rule of:

Let $p_{1}=\left(\mu_{p_{1}}, \nu_{p_{1}}\right)$ and $p_{2}=\left(\mu_{p_{2}}, \nu_{p_{2}}\right)$ be two PFNs, then:

- $s\left(p_{1}\right)<s\left(p_{2}\right) \Rightarrow p_{1} \prec p_{2}$;

- $s\left(p_{1}\right)>s\left(p_{2}\right) \Rightarrow p_{1} \succ p_{2}$;

- $s\left(p_{1}\right)=s\left(p_{2}\right) \Rightarrow p_{1} \sim p_{2}$.

It has also been pointed out by Peng and Yang [22] that the score function defined by Zhang and $\mathrm{Xu}$ [6] is not reasonable in some cases. For instance, suppose that for two PFNs, $p_{1}=(0.6,0.6)$ and $p_{2}=(0.7,0.7)$; then, by using score function (Eq. (2)), we have $p_{1} \sim$ $p_{2}$. However, $p_{1}$ and $p_{2}$ are different. Thus, with regard to the shortcoming of score function (Eq. (2)), the idea of accuracy function of a PFN has been proposed in the literature. The revised rules for comparison are as follows:
- Let $p=\left(\mu_{p}, \nu_{p}\right)$ be a PFN, then the accuracy function of $p$ is given by

$$
a(p)=\left(\mu_{p}\right)^{2}+\left(\nu_{p}\right)^{2},
$$

where $a(p)=[0,1]$. Based on the above accuracy function (Eq. (3)), the following comparison rules are provided.

- Let $p_{1}=\left(\mu_{p_{1}}, \nu_{p_{1}}\right)$ and $p_{2}=\left(\mu_{p_{2}}, \nu_{p_{2}}\right)$ be two PFNs, then

1. $s\left(p_{1}\right)<s\left(p_{2}\right) \Rightarrow p_{1} \prec p_{2}$;

2. If $s\left(p_{1}\right)=s\left(p_{2}\right)$, then:

- $a\left(p_{1}\right)<a\left(p_{2}\right) \Rightarrow p_{1} \prec p_{2}$;

- $a\left(p_{1}\right)=a\left(p_{2}\right) \Rightarrow p_{1} \sim p_{2}$.

\section{Proposed score and accuracy functions}

Here, the basics of interval-valued fuzzy numbers along with their score and accuracy functions are discussed. Also, a new accuracy function for the interval-valued fuzzy numbers is proposed and studied in contrast with the existing accuracy functions.

For an interval valued PFS $P$, consider the pair:

$$
\left(\left[\underline{\mu}_{p}(u), \bar{\mu}_{p}(u)\right],\left[\underline{\nu}_{p}(u), \bar{\nu}_{p}(u)\right]\right),
$$

as an IVPFN denoted by:

$$
p=\left(\left[\underline{\mu}_{p}(u), \bar{\mu}_{p}(u)\right],\left[\underline{\nu}_{p}(u), \bar{\nu}_{p}(u)\right]\right) .
$$

Then, for convenience, we represent IVPFN as $p=$ $\left(\left(\underline{r}_{p}, \bar{r}_{p}\right),\left(\underline{d}_{p}, \bar{d}_{q}\right)\right)$, where the pair $\left(\underline{r}_{p}, \bar{r}_{p}\right)$ is called the lower and upper strength of $p$ and the pair $\left(\underline{d}_{p}, \bar{d}_{p}\right)$ is called the lower and upper directions of the lower and upper strength of $p$, respectively. Moreover, the pairs $\left(\underline{r}_{p}, \bar{r}_{p}\right)$ and $\left(\underline{d}_{p}, \bar{d}_{p}\right)$ are connected with intervalvalued membership degree $\left[\underline{\mu}_{p}(u), \bar{\mu}_{p}(u)\right]$ and nonmembership degrees $\left[\underline{\nu}_{p}(u), \bar{\nu}_{p}(u)\right]$, indicating the support for membership/belongingness and the support against membership of $u \in P$, respectively.

The relationship between $\left(\left[\underline{\mu}_{p}(u), \bar{\mu}_{p}(u)\right],\left[\underline{\nu}_{p}(u)\right.\right.$, $\left.\left.\bar{\nu}_{p}(u)\right]\right)$ and $\left(\left(\underline{r}_{p}, \bar{r}_{p}\right),\left(\underline{d}_{p}, \bar{d}_{q}\right)\right)$ is as follows:

$$
\begin{aligned}
& \underline{\mu}_{p}(u)=\underline{r}_{p} \cos \left(\underline{\theta}_{p}\right), \underline{\nu}_{p}(u)=\underline{r}_{p} \sin \left(\underline{\theta}_{p}\right) ; \\
& \bar{\mu}_{p}(u)=\bar{r}_{p} \cos \left(\bar{\theta}_{p}\right), \bar{\nu}_{p}(u)=\bar{r}_{p} \sin \left(\bar{\theta}_{p}\right),
\end{aligned}
$$

and:

$$
\left(\underline{d}_{p}, \bar{d}_{p}\right)=\left(1-\frac{2 \underline{\theta}_{p}}{\pi}, 1-\frac{2 \bar{\theta}_{p}}{\pi}\right),
$$

where:

$$
\underline{\theta}_{p}=\arccos \left(\underline{\mu}_{p}(u) / \underline{r}_{p}\right), \quad \bar{\theta}_{p}=\arccos \left(\bar{\mu}_{p}(u) / \bar{r}_{p}\right) .
$$


Furthermore, we can easily show that the Pythagorean membership and non-membership degrees of IVPFN, $p=\left(\left[\underline{\mu}_{p}(u), \bar{\mu}_{p}(u)\right],\left[\underline{\nu}_{p}(u), \bar{\nu}_{p}(u)\right]\right)$, can be viewed as a radial length, $p=\left[\underline{r}_{p} e^{i \underline{\theta}_{p}}, \bar{r}_{p} e^{i \bar{\theta}_{p}}\right]$, in the complex plane, where:

$$
\begin{aligned}
& \underline{r}_{p}=\sqrt{\left(\underline{\mu}_{p}(u)\right)^{2}+\left(\underline{\nu}_{p}(u)\right)^{2}}, \\
& \bar{r}_{p}=\sqrt{\left(\bar{\mu}_{p}(u)\right)^{2}+\left(\bar{\nu}_{p}(u)\right)^{2}} .
\end{aligned}
$$

To resolve the issue of the comparison of IVPFNs, Peng and Yang [7] utilized the notion of score and accuracy functions, which are given as follows:

$$
\begin{aligned}
& s(p)=\frac{1}{2}\left(\left(\underline{\mu}_{p}(u)\right)^{2}+\left(\bar{\mu}_{p}(u)\right)^{2}-\left(\underline{\nu}_{p}(u)\right)^{2}-\left(\bar{\nu}_{p}(u)\right)^{2}\right), \\
& a(p)=\frac{1}{2}\left(\left(\underline{\mu}_{p}(u)\right)^{2}+\left(\bar{\mu}_{p}(u)\right)^{2}+\left(\underline{\nu}_{p}(u)\right)^{2}+\left(\bar{\nu}_{p}(u)\right)^{2}\right),
\end{aligned}
$$

where $s(p) \in[-1,1]$ and $a(p) \in[0,1]$.

Based on these functions, they provided the comparison rules of: Let $p_{1}$ and $p_{2}$ be two IVPFNs, then:

1. $s\left(p_{1}\right)<s\left(p_{2}\right) \Rightarrow p_{1} \prec p_{2}$;

2. $s\left(p_{1}\right)>s\left(p_{2}\right) \Rightarrow p_{1} \succ p_{2}$;

3. If $s\left(p_{1}\right)=s\left(p_{2}\right)$, then:

- $a\left(p_{1}\right)<a\left(p_{2}\right) \Rightarrow p_{1} \prec p_{2}$;

- $a\left(p_{1}\right)>a\left(p_{2}\right) \Rightarrow p_{1} \succ p_{2}$;

- $a\left(p_{1}\right)=a\left(p_{2}\right) \Rightarrow p_{1} \sim p_{2}$.

In some cases, it may be observed that the score and accuracy functions given by Eqs. (4) and (5) are not able to rank IVPFNs accurately. In the following example, it is shown that the existing score and the accuracy functions are not sufficiently appropriate to set the correct order of preference of the objects involved in the MCDM problem.

Example 1. Consider two IVPFNs given by:

$$
p_{1}=([0.3,0.6],[0.4,0.8]),
$$

and:

$$
p_{2}=([\sqrt{0.20}, \sqrt{0.25}],[\sqrt{0.35}, \sqrt{0.45}]) .
$$

Using Eq. (4), we get $s\left(p_{1}\right)=-0.1750$ and $s\left(p_{2}\right)=$ -0.1750. Now, we compute the value of accuracy function by using Eq. (5) and get $a\left(p_{1}\right)=0.6250$ and $a\left(p_{2}\right)=0.6250$. Therefore, based on the comparison rule, we get $p_{1} \sim p_{2}$. However, it may clearly be noted that $p_{1} \neq p_{2}$. Hence, the existing score and accuracy functions of the IVPFNs are not capable enough to give the correct order of preference.

Furthermore, to overcome the shortcomings of the accuracy function given by Eq. (5), Garg [23] proposed a new improved accuracy function by considering the hesitation degree in the formulation.

For any IVPFN, $p=\left(\left[\underline{\mu}_{p}(u), \bar{\mu}_{p}(u)\right],\left[\underline{\nu}_{p}(u), \bar{\nu}_{p}\right.\right.$ $(u)])$, the improved accuracy function $K(p)$ of $p$ is defined as:

$$
\begin{aligned}
K(p) & =\frac{1}{2}\left(\left(\underline{\mu}_{p}(u)\right)^{2}+\left(\bar{\mu}_{p}(u)\right)^{2}\right. \\
& \sqrt{1-\left(\underline{\mu}_{p}(u)\right)^{2}-\left(\underline{\nu}_{p}(u)\right)^{2}}+\left(\bar{\mu}_{p}(u)\right)^{2} \\
& \left.+\left(\underline{\mu}_{p}(u)\right)^{2} \sqrt{1-\left(\bar{\mu}_{p}(u)\right)^{2}-\left(\bar{\nu}_{p}(u)\right)^{2}}\right) .
\end{aligned}
$$

Garg [23] gave the comparison rule on the basis of Eq. (6): Let $p_{1}$ and $p_{2}$ be two IVPFNs:

- $K\left(p_{1}\right)<K\left(p_{2}\right) \Rightarrow p_{1} \prec p_{2}$;

- If $K\left(p_{1}\right)>K\left(p_{2}\right) \Rightarrow p_{1} \succ p_{2}$;

- If $K\left(p_{1}\right)=K\left(p_{2}\right) \Rightarrow p_{1} \sim p_{2}$.

If we apply Eq. (6) to Example 1, then we get $K\left(p_{1}\right)=$ 0.3809 and $K\left(p_{2}\right)=0.3636$. Since $K\left(p_{1}\right)>K\left(p_{2}\right)$, then $p_{1}$ has higher preference than $p_{2}$.

Example 2. Let $p_{1}=([0.1,0.2], \quad[\sqrt{0.05}, 0.6])$ and $p_{2}=([0.1,0.2],[\sqrt{0.04}, \sqrt{0.37}])$ be two IVPFNs; then, by using Eqs. (4) and (5), we obtain the values $s\left(p_{1}\right)=-0.1400, s\left(p_{2}\right)=-0.1400$ and $a\left(p_{1}\right)=$ $0.2700, a\left(a_{2}\right)=0.2700$. This shows that $p_{1}$ and $p_{2}$ are equivalent. However, in fact, they are different. On the other hand, by using $E q$. (6), we get $K\left(p_{1}\right)=0.04826$ and $K\left(p_{2}\right)=0.04833$, i.e. $K\left(p_{1}\right)<K\left(p_{2}\right)$, which implies that $p_{1} \prec p_{2}$. If we take the value of accuracy function up to four decimal places, then the improved accuracy function is unable to differentiate between $p_{1}$ and $p_{2}$. Hence, both existing accuracy functions (Eqs. (5) and (6)) are unable to differentiate between the IVPFNs.

Hence, to resolve the above-stated comparison issues between IVPFNs, we extend Yager's [4] accuracy function (Eq. (1)) of PFNs to IVPFNs as follows.

For each IVPFN, $p=\left(\left[\underline{r}_{p}, \bar{r}_{p}\right],\left[\underline{d}_{p}, \bar{d}_{p}\right]\right)$, we define a new accuracy function as:

$$
T(p)=\frac{2\left(\underline{r}_{p} \underline{d}_{p}+\bar{r}_{p} \bar{d}_{p}\right)-\left(\underline{r}_{p}+\bar{r}_{p}\right)+2}{4},
$$

where:

$$
\begin{aligned}
& \underline{r}_{p}=\sqrt{\left(\underline{\mu}_{p}(u)\right)^{2}+\left(\underline{\nu}_{p}(u)\right)^{2}}, \\
& \bar{r}_{p}=\sqrt{\left(\bar{\mu}_{p}(u)\right)^{2}+\left(\bar{\nu}_{p}(u)\right)^{2}},
\end{aligned}
$$


and:

$$
\left(\underline{d}_{p}, \bar{d}_{p}\right)=\left(1-\frac{2 \underline{\theta}_{p}}{\pi}, 1-\frac{2 \bar{\theta}_{p}}{\pi}\right) .
$$

Based on the proposed accuracy function (Eq. (7)), we give the comparison rules of: Let $p_{1}$ and $p_{2}$ be two IVPFNs, then:

- $T\left(p_{1}\right)<T\left(p_{2}\right) \Rightarrow p_{1} \prec p_{2}$;

- $T\left(p_{1}\right)>T\left(p_{2}\right) \Rightarrow p_{1} \succ p_{2}$;

- $T\left(p_{1}\right)=T\left(p_{2}\right) \Rightarrow p_{1} \sim p_{2}$.

If we apply the proposed accuracy function (Eq. (7)) to the above two examples, we obtain the following values:

- In Example 1, $T\left(p_{1}\right)=0.4322, T\left(p_{2}\right)=0.4288$;

- In Example 2, $T\left(p_{1}\right)=0.3782, T\left(p_{2}\right)=0.3818$;

- In light of the revised comparison rules for the proposed accuracy function (Eq. (7)), in Example 1, we have $T\left(p_{1}\right)>T\left(p_{2}\right)$, which indicate that $p_{1} \succ p_{2}$, and in Example 2, we have $T\left(p_{1}\right)<T\left(p_{2}\right)$, which indicate that $p_{1} \prec p_{2}$.

In order to demonstrate effectiveness of the proposed accuracy function, the findings and observations of this study regarding the preference order are presented in contrast with the existing accuracy functions in Table 1.

From Table 1, it is clear that for Example 1, Peng and Yang [7] score and accuracy functions are not appropriate for setting the correct preference order of IVPFNs and the preference ordering obtained using the proposed accuracy functions in this study is consistent with Garg [23] accuracy functions. For Example 2, both existing accuracy functions of Peng and Yang [7] and Garg [23] are not sufficiently appropriate for setting the correct preference order of IVPFNs, but the proposed accuracy function in this study is capable to set the correct ordering. Therefore, the ranking results obtained by the proposed accuracy function are better than those of the existing score and the accuracy functions.

Example 3. Consider two IVPNFs given by:

$$
p_{1}=([\sqrt{0.3}, \sqrt{0.6}],[\sqrt{0.2}, \sqrt{0.3}]) \text {, }
$$

and:

$$
p_{2}=([\sqrt{0.3}, \sqrt{0.4}],[\sqrt{0.1}, \sqrt{0.2}]) .
$$

We compute $\underline{r}_{p_{1}}=\sqrt{0.5}, \bar{r}_{p_{1}}=\sqrt{0.9}, \underline{d}_{p_{1}}=0.5641$, $\bar{d}_{p_{1}}=0.6082, \underline{r}_{p_{2}}=\sqrt{0.4}, \bar{r}_{p_{2}}=\sqrt{0.6}, \underline{d}_{p_{2}}=$ $0.6082, \bar{d}_{p_{2}}=0.6667, s\left(p_{1}\right)=0.2, s\left(p_{2}\right)=0.2$, and $a\left(p_{1}\right)=0.07, a\left(p_{2}\right)=0.05$. In view of the rules for comparison given in [7], we obtain $p_{1} \succ p_{2}$. Also, by the comparison rules offered by Garg [23], we get: $K\left(p_{1}\right)=0.7096, K\left(p_{2}\right)=0.5998$, i.e. $K\left(p_{1}\right)>K\left(p_{2}\right)$, which implies that $p_{1} \succ p_{2}$. However, by Eq. (7), we get $T\left(p_{1}\right)=0.5740, T\left(p_{2}\right)=0.5946$, i.e., $T\left(p_{1}\right)<T\left(p_{2}\right)$ which implies that $p_{1} \prec p_{2}$. Hence, the comparison rules of the proposed accuracy function (Eq. (7)), lead to contradictory results.

In the above example, the direction of commitment $\underline{d}_{p_{1}}(>\pi / 4)$ is greater than the direction of commitment $\bar{d}_{p_{2}}(<\pi / 4)$ and $\bar{d}_{p_{1}}=\underline{d}_{p_{2}}=0.6082<$ $\pi / 4$, which indicates that $p_{2}$ supports the membership better than $p_{1}$, i.e., $p_{2}$ is preferred to $p_{1}$ (refer to Table 2). Since the proposed accuracy function (Eq. (7)) takes the direction of commitment of IVPFN as an input, its ranking result may be better than the existing

\begin{tabular}{|c|c|c|c|c|}
\hline \multirow{4}{*}{ Example 1} & Methodology & \multicolumn{2}{|c|}{ Score and accuracy functions } & \multirow{2}{*}{$\begin{array}{c}\text { Preference order } \\
p_{1} \sim p_{2}\end{array}$} \\
\hline & Peng and Yang [7] & $\begin{array}{l}s\left(p_{1}\right)=-0.175 \\
a\left(p_{1}\right)=0.625\end{array}$ & $\begin{array}{l}s\left(p_{2}\right)=-0.17 \\
a\left(p_{2}\right)=0.625\end{array}$ & \\
\hline & Garg [23] & $K\left(p_{1}\right)=0.381$ & $K\left(p_{2}\right)=0.364$ & $p_{1} \succ p_{2}$ \\
\hline & Proposed method & $T\left(p_{1}\right)=0.432$ & $T\left(p_{2}\right)=0.429$ & $p_{1} \succ p_{2}$ \\
\hline \multirow{3}{*}{ Example 2} & Peng and Yang [7] & $\begin{array}{l}s\left(p_{1}\right)=-0.140 \\
a\left(p_{1}\right)=0.270\end{array}$ & $\begin{array}{l}s\left(p_{2}\right)=-0.140 \\
a\left(p_{2}\right)=0.270\end{array}$ & $p_{1} \sim p_{2}$ \\
\hline & Garg [23] & $K\left(p_{1}\right)=0.0483$ & $K\left(p_{2}\right)=0.0483$ & $p_{1} \sim p_{2}$ \\
\hline & Proposed method & $T\left(p_{1}\right)=0.378$ & $T\left(p_{2}\right)=0.382$ & $p_{1} \prec p_{2}$ \\
\hline
\end{tabular}
score and the accuracy functions.

Table 1. Comparative analysis for Examples 1 and 2. 
Table 2. Comparative analysis for Example 3.

\begin{tabular}{ccccc}
\hline & Methodology & Score and accuracy functions & Preference order \\
\cline { 2 - 4 } & Peng and Yang $[7]$ & $s\left(p_{1}\right)=0.2$, & $s\left(p_{2}\right)=0.2$, & \\
& $a\left(p_{1}\right)=0.07$, & $a\left(p_{2}\right)=0.05$ & \\
& & & \\
Example 3 & Garg [23] & $K\left(p_{1}\right)=0.7096$, & $K\left(p_{2}\right)=0.5998$ & $p_{1} \succ p_{2}$ \\
& & & & \\
& Proposed method & $T\left(p_{1}\right)=0.5740$, & $T\left(p_{2}\right)=0.5946$ & $p_{1} \prec p_{2}$ \\
\hline
\end{tabular}

\section{IVPF distance measures and weighted averaging operators}

In this section, first, we present the distance measures and weighted averaging operators proposed by Liu et al. [21] for IVPFNs. Then, a new distance measure is given for IVFFNs and the shortcomings in the existing distance measure proposed by Liu et al. [21] are discussed.

Liu et al. [21] proposed the following IVPF $p$-distance measure, generalized IVPF weighted distance measure, generalized probabilistic IVPF Ordered Weighted Averaging (OWA) distance operators, and further proceedings for IVPFNs.

Definition 1. IVPF $\boldsymbol{p}$-distance measure: Consider any two IVPFNs:

$$
\xi_{1}=\left(\mu_{\xi_{1}}, \nu_{\xi_{1}}\right)=\left(\left[\underline{\mu}_{\xi_{1}}, \bar{\mu}_{\xi_{1}}\right],\left[\underline{\nu}_{\xi_{1}}, \bar{\nu}_{\xi_{1}}\right]\right),
$$

and:

$$
\xi_{2}=\left(\mu_{\xi_{2}}, \nu_{\xi_{2}}\right)=\left(\left[\underline{\mu}_{\xi_{2}}, \bar{\mu}_{\xi_{2}}\right],\left[\underline{\nu}_{\xi_{2}}, \bar{\nu}_{\xi_{2}}\right]\right) .
$$

The IVPF p-distance between $\xi_{1}$ and $\xi_{2}$ is denoted by $d_{p}\left(\xi_{1}, \xi_{2}\right)$ and defined as follows:

$$
\begin{aligned}
d^{p}\left(\xi_{1}, \xi_{2}\right)= & \frac{1}{4}\left[\left|\left(\underline{\mu}_{\xi_{1}}\right)^{2}-\left(\underline{\mu}_{\xi_{2}}\right)^{2}\right|^{p}+\left|\left(\bar{\mu}_{\xi_{1}}\right)^{2}-\left(\bar{\mu}_{\xi_{2}}\right)^{2}\right|^{p}\right. \\
& +\left|\left(\underline{\nu}_{\xi_{1}}\right)^{2}-\left(\underline{\nu}_{\xi_{2}}\right)^{2}\right|^{p}+\left|\left(\bar{\nu}_{\xi_{1}}\right)^{2}-\left(\bar{\nu}_{\xi_{2}}\right)^{2}\right|^{p} \\
& +\left|\left(\underline{\pi}_{\xi_{1}}\right)^{2}-\left(\underline{\pi}_{\xi_{2}}\right)^{2}\right|^{p}+\mid\left(\bar{\pi}_{\xi_{1}}\right)^{2} \\
& \left.-\left.\left(\bar{\pi}_{\xi_{2}}\right)^{2}\right|^{p}\right] .
\end{aligned}
$$

Definition 2. Generalized IVPF Weighted Distance measure: Let $A=\left(\xi_{1}, \xi_{2}, \ldots, \xi_{n}\right)$ and $B=$ $\left(\eta_{1}, \eta_{2}, \ldots, \eta_{n}\right)$ be two n-tuples of IVPFNs, where $\left(\mu_{\xi_{i}}, \nu_{\xi_{i}}\right)=\left(\left[\underline{\mu}_{\xi_{i}}, \bar{\mu}_{\xi_{i}}\right],\left[\underline{\nu}_{\xi_{i}}, \bar{\nu}_{\xi_{i}}\right]\right)$ and $\left(\mu_{\eta_{i}}, \nu_{\eta_{i}}\right)=$ $\left(\left[\underline{\mu}_{\eta_{i}}, \bar{\mu}_{\eta_{i}}\right],\left[\underline{\nu}_{\eta_{i}}, \bar{\nu}_{\eta_{i}}\right]\right)$. Then, the generalized IVPF Weighted Distance (GIVPFWD) measure is a function of $\Phi: I V P F N^{n} \times I V P F N^{n} \rightarrow \mathbb{R}$ and defined as:

$$
\Phi(A, B)=\left(\sum_{i=1}^{n} \omega_{i} d^{p}\left(\xi_{i}, \eta_{i}\right)\right)^{1 / p},
$$

where $\omega=\left(\omega_{1}, \omega_{2}, \ldots, \omega_{n}\right)^{T}$ is a weight vector with $\omega_{i}>0, \sum_{i=1}^{n} \omega_{i}=1$ and $d^{p}\left(\xi_{i}, \eta_{i}\right)$ is the IVPF p-distance between IVPFNs $\xi_{i}$ and $\eta_{i}$ defined by Eq. (8).

In addition, the following points should be mentioned:

- If $d^{p}\left(\xi_{i}, \eta_{i}\right)$ is the $i$ th largest value of $d^{p}\left(\xi_{j}, \eta_{j}\right), j=$ $1,2, \ldots, n$ in Eq. (9), then the distance measure (Eq. (9)) is called the generalized IVPF ordered weighted distance (GIVPFOWD) measure for IVPFNs.

- If we take $p=1$ in Eq. (9), then it becomes the IVPF Weighted Averaging Distance (IVPFWAD) measure given by:

$$
\Phi(A, B)=\sum_{i=1}^{n} \omega_{i} d^{1}\left(\xi_{i}, \eta_{i}\right) .
$$

- If we take $p=2$ in Eq. (9), then it becomes the IVPF Weighted Euclidean Distance (IVPFWED) measure given by:

$$
\Phi(A, B)=\left(\sum_{i=1}^{n} \omega_{i} d^{2}\left(\xi_{i}, \eta_{i}\right)\right)^{1 / 2} .
$$

Definition 3. Generalized probabilistic IVPFOWA distance operators. Let $A=\left(\xi_{1}, \xi_{2}, \ldots, \xi_{n}\right)$ and $B=\left(\eta_{1}, \eta_{2}, \ldots, \eta_{n}\right)$ be two $n$-tuples of IVPFNs, where

$$
\left(\mu_{\xi_{i}}, \nu_{\xi_{i}}\right)=\left(\left[\underline{\mu}_{\xi_{i}}, \bar{\mu}_{\xi_{i}}\right],\left[\underline{\nu}_{\xi_{i}}, \bar{\nu}_{\xi_{i}}\right]\right)
$$

and

$$
\left(\mu_{\eta_{i}}, \nu_{\eta_{i}}\right)=\left(\left[\underline{\mu}_{\eta_{i}}, \bar{\mu}_{\eta_{i}}\right],\left[\underline{\nu}_{\eta_{i}}, \bar{\nu}_{\eta_{i}}\right]\right) .
$$

Then, the Probabilistic generalized IVPF Weighted Averaging Distance (P-GIVPFWAD) operator is a function of $\Phi: I V P F N^{n} \times I V P F N^{n} \rightarrow \mathbb{R}$ and defined as: 


$$
\Phi(A, B)=\left(\sum_{i=1}^{n} \rho_{i} d^{p}\left(\xi_{i}, \eta_{i}\right)\right)^{1 / p},
$$

where $\omega=\left(\omega_{1}, \omega_{2}, \ldots, \omega_{n}\right)^{T}$ is a weight vector with $\omega_{i}>0, \sum_{i=1}^{n} \omega_{i}=1 ; \rho_{i}=\lambda_{i} w_{i}+\left(1-\lambda_{i}\right) p_{i}$ and $p_{i}$ is the associated probability of IVPF $p$-distance $d^{p}\left(\xi_{i}, \eta_{i}\right)$. $\lambda_{i} \in[0,1]$ and $\left(1-\lambda_{i}\right)$ represent the degree of weight and the degree of probabilistic information, respectively.

\section{Remarks}

- If $\lambda_{i}=0$, then GPWIVPF distance measure (Eq. (12)) is called the probabilistic generalized IVPF distance measure.

- If $\lambda_{i}=1$, then it reduces to GIVPFWD distance measure (Eq. (9)).

- If we take $p=1$ in Eq. (12), then it becomes PIVPFWAD operator, given by:

$$
\Phi(A, B)=\sum_{i=1}^{n} \rho_{i} d^{1}\left(\xi_{i}, \eta_{i}\right) .
$$

- If we take $p=2$ in Eq. (12), then it becomes the Probabilistic Interval-Valued Pythagorean Fuzzy Weighted Euclidean Distance (P-IVPFWED) operator, given by:

$$
\Phi(A, B)=\left(\sum_{i=1}^{n} \rho_{i} d^{2}\left(\xi_{i}, \eta_{i}\right)\right)^{1 / 2} .
$$

- If $d^{p}\left(\xi_{i}, \eta_{i}\right)$ is the $i$ th largest $d^{p}\left(\xi_{j}, \eta_{j}\right), j=$ $1,2, \ldots, n$ and $p_{i}$ is its corresponding probability then the distance measure (Eq. (12)) is called PGIVPFOWAD operator.

- If we take $\rho_{i}=\frac{w_{i} p_{i}}{\sum_{i=1}^{n} w_{i} p_{i}}$ and $d^{p}\left(\xi_{i}, \eta_{i}\right)$ as the $i$ th largest $d^{p}\left(\xi_{j}, \eta_{j}\right), j=1,2, \ldots, n$ with $p_{i}$ as the corresponding probability of the $i$ th largest $d^{p}\left(\xi_{j}, \eta_{j}\right)$, then the distance measure (Eq. (12)) is called IPGIVPFOWAD operator.

It may be observed that the above distance measures or operators sometimes lead to some unreasonable output. For instance:

Example 4. Let $\xi_{1}=([0.5,0.6],[0.6,0.7]), \xi_{2}=$ $([0.6,0.7],[0.5,0.6])$ and $\xi_{3}=([0.3,0.4],[0.4,0.5])$ be three IVPFNs then, by using Eq. (8) for $p=1$, we have:

$$
\begin{aligned}
& d\left(\xi_{1}, \xi_{3}\right)=d\left(\xi_{2}, \xi_{3}\right)=0.40 \\
& \underline{r}_{\xi_{1}}=\underline{r}_{\xi_{2}}=0.7810 ; \quad \bar{r}_{\xi_{1}}=\bar{r}_{\xi_{2}}=0.9220, \\
& \underline{\theta}_{\xi_{1}}=0.8761, \quad \bar{\theta}_{\xi_{1}}=0.8622 ; \\
& \underline{\theta}_{\xi_{2}}=0.6947, \quad \bar{\theta}_{\xi_{2}}=0.7086 ; \\
& \underline{d}_{\xi_{1}}=0.4423, \quad \bar{d}_{\xi_{1}}=0.4511 ; \\
& \underline{d}_{\xi_{2}}=0.5577, \quad \bar{d}_{\xi_{2}}=0.5489 .
\end{aligned}
$$

It is observed that $\xi_{1}$ and $\xi_{2}$ have the same lower and upper lengths, but different lower and upper directions of commitment (strength). Thus, the distance between $\xi_{1}$ and $\xi_{3}$ should be different from the distance between $\xi_{2}$ and $\xi_{3}$. Hence, in this case IVPF p-distance measure (Eq. (8)) is not appropriate to use.

Since the strength and direction of commitment are important parameters of IVPFNs, ignoring them may lead to inappropriate results. Hence, by taking all the four parameters into account for IVPFNs,

$$
\left[\underline{\mu}_{p}(u), \bar{\mu}_{p}(u)\right],\left[\underline{\nu}_{p}(u), \bar{\nu}_{p}(u)\right],\left(\underline{r}_{p}, \bar{r}_{p}\right) \text {, and }\left(\underline{d}_{p}, \bar{d}_{p}\right) \text {, }
$$

we propose the IVPF $p$-distance measure between two IVPFNs, $\xi_{1}$ and $\xi_{2}$, as follows:

$$
\begin{aligned}
d_{T}^{p}\left(\xi_{1}, \xi_{2}\right)= & \frac{1}{4}\left[\left|\underline{\mu}_{\xi_{1}}-\underline{\mu}_{\xi_{2}}\right|^{p}+\left|\bar{\mu}_{\xi_{1}}-\bar{\mu}_{\xi_{2}}\right|^{p}\right. \\
& +\left|\underline{\nu}_{\xi_{1}}-\underline{\nu}_{\xi_{2}}\right|^{p}+\left|\bar{\nu}_{\xi_{1}}-\bar{\nu}_{\xi_{2}}\right|^{p} \\
& +\left|\underline{r}_{\xi_{1}}-\underline{r}_{\xi_{2}}\right|^{p}+\left|\bar{r}_{\xi_{1}}-\bar{r}_{\xi_{2}}\right|^{p} \\
& \left.+\left|\underline{d}_{\xi_{1}}-\underline{d}_{\xi_{2}}\right|^{p}+\left|\bar{d}_{\xi_{1}}-\bar{d}_{\xi_{2}}\right|^{p}\right] .
\end{aligned}
$$

By using the proposed IVPF $p$-distance measure (Eq. (15)) for $p=1$, for Example 4, we have $d_{T}\left(\xi_{1}, \xi_{3}\right)=0.3542$ and $d_{T}\left(\xi_{2}, \xi_{3}\right)=0.4075$.

Based on Table 3 , we can state that the proposed IVPF $p$-distance measure (Eq. (15)) is a better measure than Eq. (8).

\section{Application to a MCDM problem}

In this section, we employ the proposed accuracy function, distance measures, and OWA operators to deal with MCDM problem with interval-valued

Table 3. Comparative analysis for Example 4.

\begin{tabular}{lll}
\hline & Methodology & IVPF $\boldsymbol{p}$-distance measure \\
\cline { 2 - 3 } Example 4 & Liu et al. [21] & $d\left(\xi_{1}, \xi_{3}\right)=d\left(\xi_{2}, \xi_{3}\right)=0.40$ \\
& Proposed method & $d_{T}\left(\xi_{1}, \xi_{3}\right)=0.3542, d_{T}\left(\xi_{2}, \xi_{3}\right)=0.4075$ \\
\hline
\end{tabular}


Pythagorean fuzzy information. Consider the following setup of an MCDM problem in IVPFNs environment.

Consider the set of $m$ possible alternatives, say, $X=\left\{x_{1}, x_{2}, \ldots, x_{m}\right\}$, and the set of $n$-criteria by which the performance of the alternatives is evaluated, say, $C=\left\{C_{1}, C_{2}, \ldots, C_{n}\right\}$. Let $\omega=\left\{\omega_{1}, \omega_{2}, \ldots, \omega_{n}\right\}$ be the weight vector of all criteria such that $0 \leq \omega_{i} \leq 1$ and $\sum_{i=1}^{n} \omega_{i}=1$. Assume that the performance of an alternative $x_{i}(i=1,2, \ldots, m)$ with respect to the criteria $C_{j}, j=1,2, \ldots, n$ is measured by IVPFNs $C_{j}\left(x_{i}\right)=\left(\left[\mu_{i, j}, \mu_{i, j}\right],\left[\nu_{i, j}, \nu_{i, j}\right]\right), j=1,2, \ldots, n ; i=$ $1,2 \ldots, m$; here, $\left[\mu_{i, j}, \mu_{i, j}\right]$ represents the degree that alternative $x_{i}$ satisfies the criterion $C_{j}$ and $\left[\nu_{i, j}, \nu_{i, j}\right]$ represents the degree that alternative $x_{i}$ does not satisfy the criterion $C_{j}$. Let $D_{m \times n}=C_{j}\left(x_{i}\right)_{m \times n}$ be an interval-valued Pythagorean fuzzy decision matrix. The procedural steps of the proposed MCDM algorithm are as follows:

Step 1: Compute the accuracy of each IVPFN of the obtained decision matrix $M_{m \times n}=C_{j}\left(x_{i}\right)_{m \times n}$ by applying the proposed accuracy function (Eq. (7)).

Step 2: Determine the IVPF-Positive Ideal Solution (PIS):

$$
x^{+}=\left\{\left\langle x_{i}, \max _{i} T\left(C_{j}\left(x_{i}\right)\right)\right\rangle\right\},
$$

and the IVPF-Negative Ideal Solution (NIS):

$$
x^{-}=\left\{\left\langle x_{i}, \min _{i} T\left(C_{j}\left(x_{i}\right)\right)\right\rangle\right\}
$$

for $j=1,2, \ldots, n ; i=1,2, \ldots, m$ with the help of the accuracies of IVPFNs obtained in Step 1.

Step 3: Evaluate the distance of each alternative $x_{i}, i=1,2, \ldots, m$ from $x^{+}$and $x^{-}$using the proposed IVPF $p$-distance measure (Eq. (15)).

Step 4: Using the values obtained in Step 3 and rearranging the probability weight, evaluate the new weights by using:

$$
\rho_{i}=\lambda_{i} w_{i}+\left(1-\lambda_{i}\right) p_{i} \quad \text { or } \quad \rho_{i}=\frac{w_{i} p_{i}}{\sum_{i=1}^{n} w_{i} p_{i}} .
$$

Step 5: Determine the P-GIVPFOWAD or IPGIVPFOWAD of the alternative $x_{i}$ by the positive ideal IVPFN solution $x^{+}$and the negative ideal IVPFN solution $x^{-}$.

Step 6: Compute the coefficient of relative closeness for each alternative $x_{i}$ as follows:

$$
\begin{aligned}
& r\left(x_{i}\right)=\frac{D\left(x_{i}, x^{-}\right)}{D\left(x_{i}, x^{+}\right)+D\left(x_{i}, x^{-}\right)}, \\
& i=1,2, \ldots, m,
\end{aligned}
$$

where $D(\cdot)$ is an IP-GIVPFOWAD or PGIVPFOWAD.
Step 7: Rank all the alternatives based on the coefficient of relative closeness $r\left(x_{i}\right)$ and choose the optimal alternative.

\subsection{Numerical example}

In order to illustrate the implementation of the steps of the proposed algorithm stated above, we consider the following MCDM problem of the selection of the strategy for an optimal production referring to the related literature and undertakings completed in [21,24,25].

Suppose that a firm desires to manufacture a new product and looks for the optimal target of having the maximum benefit. Based upon a survey analysis of the market, they lay down the following five possible strategies (alternatives):

- $x_{1}$ : Creating a new product aligned with rich customers;

- $x_{2}$ : Creating a new product aligned with mid-level customers;

- $x_{3}$ : Creating a new product aligned with low-level customers;

- $x_{4}$ : Creating a new product suited to all customers;

- $x_{5}$ : No manufacturing of any product.

After a detailed investigation into the information received from sources, the decision makers go for the following general criteria for the adaptability of strategies to production:

- $C_{1}$ : Short-term benefits;

- $C_{2}$ : Mid-term benefits;

- $C_{3}$ : Long-term benefits;

- $C_{4}$ : Production risk;

- $C_{5}$ : Various other factors.

Construct the decision matrix:

$$
\begin{aligned}
& D_{5 \times 5}=C_{j}\left(x_{i}\right)_{5 \times 5}=\left(\left[\mu_{i, j}, \mu_{i, j}\right],\left[\nu_{i, j}, \nu_{i, j}\right]\right), \\
& j=1,2, \ldots, 5 ; \quad i=1,2, \ldots, 5,
\end{aligned}
$$

as shown in Table 4.

Suppose that with reference to the problem under consideration, the decision makers find the probabilistic information $p=(0.3,0.3,0.2,0.1,0.1)$ and weight vector $w=(0.2,0.25,0.15,0.3,0.1)$, which represents the degree of importance/weightage of each criterion. Then, to get the most desirable alternative, we apply the steps of the proposed algorithm. First, we compute the accuracy of each IVPFN of the decision matrix as shown in Table 4 by applying the proposed accuracy function (Eq. (7)). The computed values are tabulated in Table 5. 
Table 4. Interval-valued Pythagorean fuzzy decision matrix.

\begin{tabular}{cccccc}
\hline & $\boldsymbol{C}_{\mathbf{1}}$ & $\boldsymbol{C}_{\mathbf{2}}$ & $\boldsymbol{C}_{\boldsymbol{3}}$ & $\boldsymbol{C}_{4}$ & $\boldsymbol{C}_{\mathbf{5}}$ \\
\hline $\boldsymbol{x}_{\mathbf{1}}$ & $([0.6,0.7],[0.5,0.6])$ & $([0.4,0.5],[0.5,0.6])$ & $([0.2,0.6],[0.3,0.6])$ & $([0.5,0.6],[0.4,0.5])$ & $([0.2,0.7],[0.3,0.7])$ \\
$\boldsymbol{x}_{\mathbf{2}}$ & $([0.5,0.6],[0.6,0.7])$ & $([0.5,0.6],[0.4,0.5])$ & $([0.3,0.6],[0.2,0.6])$ & $([0.4,0.5],[0.5,0.6])$ & $([0.3,0.7],[0.2,0.7])$ \\
$\boldsymbol{x}_{\mathbf{3}}$ & $([0.3,0.6],[0.4,0.8])$ & $([0.6,0.7],[0.4,0.5])$ & $([0.6,0.8],[0.3,0.4])$ & $([0.4,0.5],[0.3,0.6])$ & $([0.3,0.4],[0.7,0.8])$ \\
$\boldsymbol{x}_{4}$ & $([0.5,0.7],[0.5,0.6])$ & $([0.4,0.5],[0.1,0.3])$ & $([0.1,0.2],[0.4,0.6])$ & $([0.4,0.5],[0.1,0.2])$ & $([0.5,0.7],[0.4,0.6])$ \\
$\boldsymbol{x}_{\mathbf{5}}$ & $([0.3,0.4],[0.1,0.2])$ & $([0.4,0.6],[0.4,0.5])$ & $([0.2,0.3],[0.5,0.6])$ & $([0.4,0.6],[0.5,0.6])$ & $([0.6,0.8],[0.3,0.4])$ \\
\hline
\end{tabular}

Table 5. Results of applying accuracy function.

\begin{tabular}{cccccc}
\hline & $\boldsymbol{C}_{\boldsymbol{1}}$ & $\boldsymbol{C}_{\boldsymbol{2}}$ & $\boldsymbol{C}_{\boldsymbol{3}}$ & $\boldsymbol{C}_{\boldsymbol{4}}$ & $\boldsymbol{C}_{\mathbf{5}}$ \\
\hline $\boldsymbol{x}_{\boldsymbol{1}}$ & 0.5451 & 0.4549 & 0.4773 & 0.5451 & 0.4773 \\
$\boldsymbol{x}_{\boldsymbol{2}}$ & 0.4549 & 0.5451 & 0.5227 & 0.4549 & 0.5227 \\
$\boldsymbol{x}_{\boldsymbol{3}}$ & 0.4322 & 0.5905 & 0.6603 & 0.5000 & 0.3162 \\
$\boldsymbol{x}_{\boldsymbol{4}}$ & 0.5225 & 0.6164 & 0.3357 & 0.6403 & 0.5451 \\
$\boldsymbol{x}_{\boldsymbol{5}}$ & 0.5925 & 0.5225 & 0.3619 & 0.4774 & 0.6603 \\
\hline
\end{tabular}

Based on the accuracies obtained in Table 5, we find the IVPF-PIS $x^{+}$and the IVPF-NIS $x^{-}$, respectively, as follows:

$$
\begin{aligned}
x^{+}= & \left\{\left\langle C_{1},([0.3,0.4],[0.1,0.2])\right\rangle,\right. \\
& \left\langle C_{2},([0.4,0.5],[0.1,0.3])\right\rangle, \\
& \left\langle C_{3},([0.6,0.8],[0.3,0.4])\right\rangle, \\
& \left\langle C_{4},([0.4,0.5],[0.1,0.2])\right\rangle, \\
& \left.\left\langle C_{5},([0.6,0.8],[0.3,0.4])\right\rangle\right\},
\end{aligned}
$$

and:

$$
\begin{aligned}
x^{-}= & \left\{\left\langle C_{1},([0.3,0.6],[0.4,0.8])\right\rangle,\right. \\
& \left\langle C_{2},([0.4,0.5],[0.5,0.6])\right\rangle, \\
& \left\langle C_{3},([0.1,0.2],[0.4,0.6])\right\rangle, \\
& \left\langle C_{4},([0.4,0.5],[0.5,0.6])\right\rangle, \\
& \left.\left\langle C_{5},([0.3,0.4],[0.7,0.8])\right\rangle\right\} .
\end{aligned}
$$

Furthermore, we evaluate the P-GIVPFOWAD or IPGIVPFOWAD for each alternative $x_{i}, i=1,2, \ldots, m$ from the IVPF-PIS $x^{+}$and the IVPF-NIS $x^{-}$. The respective results are shown in Tables 6 to 9 . Then, we find the relative closeness coefficient by Eq. (16) for each alternative $x_{i}$. The results are given in Tables 10 and 11 and illustrated in Figures 1 and 2. In Tables 10 to 12 , we observe that for different values of parameter $p$, the ranking order of alternatives remains unchanged by applying either P-GIVPFOWAD or IPGIVPFOWAD and all the results show that $x_{5}$ is the optimal alternative.

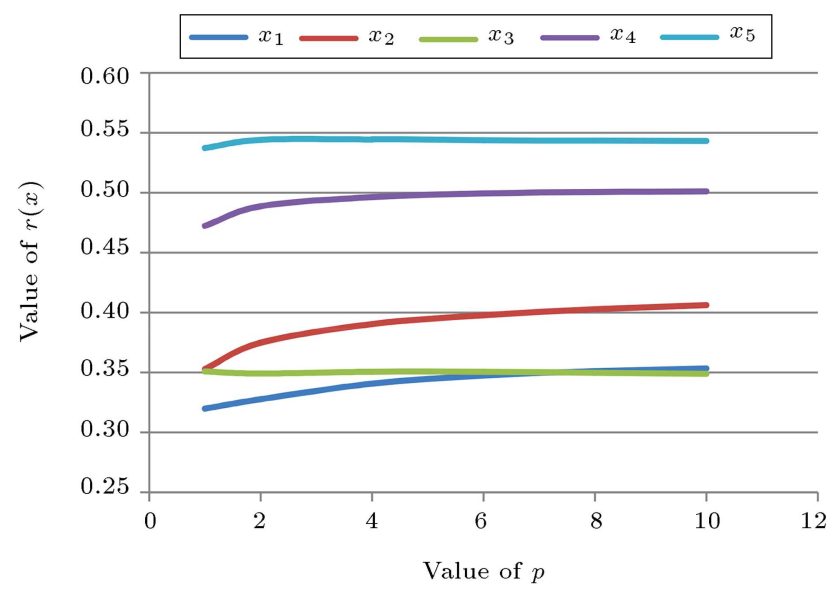

Figure 1. Ranking order based on P-GIVPFOWAD.

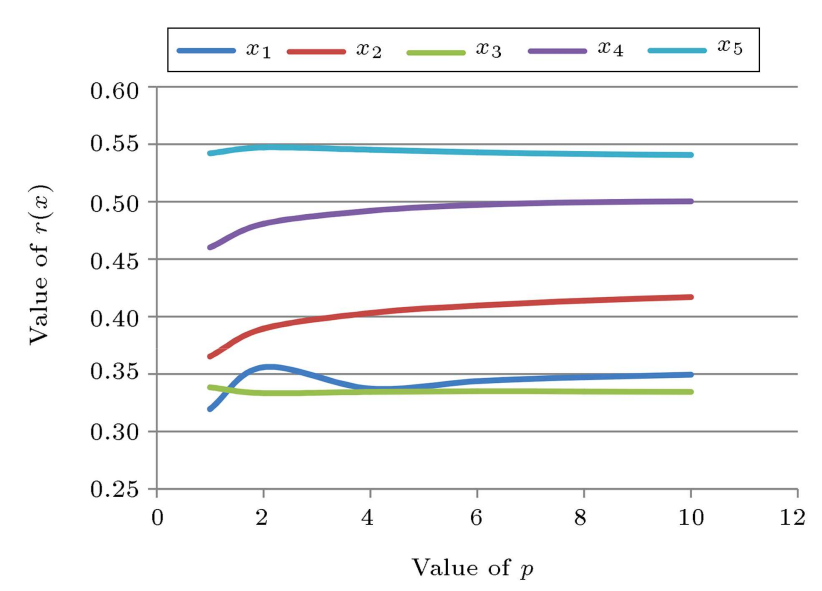

Figure 2. Ranking order based on IP-GIVPFOWAD.

\section{Remarks on limitations of existing methods}

Based on the numerical example and the values obtained in the previous section, we put forward some remarks on the limitations of the existing methods:

- If we deal with the MCDM problem with the proposed accuracy function (Eq. (7)) and Liu et al.'s [21] distance measure (Eq. (8)), Based on Table 9 , we observe that the distances of $x_{1}$ and $x_{2}$ from IVPF-PIS $x^{+}$for $p=1$ are the same. However, the alternatives $x_{1}$ and $x_{2}$ with respect to all criteria $C_{j}, j=1,2, \ldots, n$, take different IVPFNs; 
Table 6. Distances between $x_{i}$ and $x^{+}$obtained by P-GIVPFOWAD.

\begin{tabular}{cccccc}
\hline Value of $\boldsymbol{p}$ & $\boldsymbol{D}\left(\boldsymbol{x}_{\boldsymbol{1}}, \boldsymbol{x}^{+}\right)$ & $\boldsymbol{D}\left(\boldsymbol{x}_{\boldsymbol{2}}, \boldsymbol{x}^{+}\right)$ & $\boldsymbol{D}\left(\boldsymbol{x}_{\boldsymbol{3}}, \boldsymbol{x}^{+}\right)$ & $\boldsymbol{D}\left(\boldsymbol{x}_{\boldsymbol{4}}, \boldsymbol{x}^{+}\right)$ & $\boldsymbol{D}\left(\boldsymbol{x}_{\mathbf{5}}, \boldsymbol{x}^{+}\right)$ \\
\hline 1 & 0.4934 & 0.4783 & 0.4152 & 0.3468 & 0.2807 \\
2 & 0.3924 & 0.3717 & 0.3331 & 0.2635 & 0.2234 \\
4 & 0.3681 & 0.3462 & 0.3138 & 0.2421 & 0.2125 \\
6 & 0.3690 & 0.3452 & 0.3150 & 0.2405 & 0.2145 \\
8 & 0.3728 & 0.3470 & 0.3183 & 0.2417 & 0.2171 \\
10 & 0.3766 & 0.3490 & 0.3214 & 0.2434 & 0.2194 \\
\hline
\end{tabular}

Table 7. Distances between $x_{i}$ and $x^{-}$obtained by P-GIVPFOWAD

\begin{tabular}{cccccc}
\hline Value of $\boldsymbol{p}$ & $\boldsymbol{D}\left(\boldsymbol{x}_{\boldsymbol{1}}, \boldsymbol{x}^{-}\right)$ & $\boldsymbol{D}\left(\boldsymbol{x}_{\boldsymbol{2}}, \boldsymbol{x}^{-}\right)$ & $\boldsymbol{D}\left(\boldsymbol{x}_{\boldsymbol{3}}, \boldsymbol{x}^{-}\right)$ & $\boldsymbol{D}\left(\boldsymbol{x}_{\boldsymbol{4}}, \boldsymbol{x}^{-}\right)$ & $\boldsymbol{D}\left(\boldsymbol{x}_{\mathbf{5}}, \boldsymbol{x}^{-}\right)$ \\
\hline 1 & 0.2320 & 0.2609 & 0.2245 & 0.3103 & 0.3258 \\
2 & 0.1912 & 0.2229 & 0.1787 & 0.2519 & 0.2666 \\
4 & 0.1902 & 0.2217 & 0.1695 & 0.2385 & 0.2539 \\
6 & 0.1965 & 0.2280 & 0.1700 & 0.2398 & 0.2555 \\
8 & 0.2017 & 0.2339 & 0.1712 & 0.2423 & 0.2583 \\
10 & 0.2057 & 0.2386 & 0.1723 & 0.2444 & 0.2607 \\
\hline
\end{tabular}

Table 8. Distances between $x_{i}$ and $x^{+}$obtained by IP-GIVPFOWAD.

\begin{tabular}{cccccc}
\hline Value of $\boldsymbol{p}$ & $\boldsymbol{D}\left(\boldsymbol{x}_{\mathbf{1}}, \boldsymbol{x}^{+}\right)$ & $\boldsymbol{D}\left(\boldsymbol{x}_{\boldsymbol{2}}, \boldsymbol{x}^{+}\right)$ & $\boldsymbol{D}\left(\boldsymbol{x}_{\boldsymbol{3}}, \boldsymbol{x}^{+}\right)$ & $\boldsymbol{D}\left(\boldsymbol{x}_{\boldsymbol{4}}, \boldsymbol{x}^{+}\right)$ & $\boldsymbol{D}\left(\boldsymbol{x}_{\boldsymbol{5}}, \boldsymbol{x}^{+}\right)$ \\
\hline 1 & 0.4715 & 0.4568 & 0.3903 & 0.3662 & 0.2453 \\
2 & 0.3815 & 0.3510 & 0.3122 & 0.2750 & 0.1963 \\
4 & 0.3619 & 0.3281 & 0.2918 & 0.2501 & 0.1871 \\
6 & 0.3647 & 0.3279 & 0.2910 & 0.2475 & 0.1889 \\
8 & 0.3694 & 0.3301 & 0.2929 & 0.2483 & 0.1914 \\
10 & 0.3738 & 0.3325 & 0.2951 & 0.2499 & 0.1935 \\
\hline
\end{tabular}

Table 9. Distances between $x_{i}$ and $x^{-}$obtained by IP-GIVPFOWAD.

\begin{tabular}{cccccc}
\hline Value of $\boldsymbol{p}$ & $\boldsymbol{D}\left(\boldsymbol{x}_{\boldsymbol{1}}, \boldsymbol{x}^{-}\right)$ & $\boldsymbol{D}\left(\boldsymbol{x}_{\boldsymbol{2}}, \boldsymbol{x}^{-}\right)$ & $\boldsymbol{D}\left(\boldsymbol{x}_{\boldsymbol{3}}, \boldsymbol{x}^{-}\right)$ & $\boldsymbol{D}\left(\boldsymbol{x}_{\boldsymbol{4}}, \boldsymbol{x}^{-}\right)$ & $\boldsymbol{D}\left(\boldsymbol{x}_{\mathbf{5}}, \boldsymbol{x}^{-}\right)$ \\
\hline 1 & 0.2213 & 0.2629 & 0.1995 & 0.3122 & 0.2902 \\
2 & 0.1842 & 0.2238 & 0.1561 & 0.2545 & 0.2372 \\
4 & 0.1843 & 0.2215 & 0.1466 & 0.2423 & 0.2241 \\
6 & 0.1910 & 0.2275 & 0.1466 & 0.2445 & 0.2243 \\
8 & 0.1965 & 0.2331 & 0.1475 & 0.2476 & 0.2260 \\
10 & 0.2008 & 0.2377 & 0.1483 & 0.2501 & 0.2277 \\
\hline
\end{tabular}

Table 10. Relative closeness coefficients obtained by P-GIVPFOWAD.

\begin{tabular}{ccccccc}
\hline Value of $\boldsymbol{p}$ & $\boldsymbol{r}\left(\boldsymbol{x}_{\mathbf{1}}\right)$ & $\boldsymbol{r}\left(\boldsymbol{x}_{\mathbf{2}}\right)$ & $\boldsymbol{r}\left(\boldsymbol{x}_{\mathbf{3}}\right)$ & $\boldsymbol{r}\left(\boldsymbol{x}_{\mathbf{4}}\right)$ & $\boldsymbol{r}\left(\boldsymbol{x}_{\mathbf{5}}\right)$ & Ranking order \\
\hline 1 & 0.3198 & 0.3529 & 0.3509 & 0.4722 & 0.5372 & $x_{5} \unrhd x_{4} \unrhd x_{2} \unrhd x_{3} \unrhd x_{1}$ \\
2 & 0.3276 & 0.3749 & 0.3492 & 0.4887 & 0.5441 & $x_{5} \unrhd x_{4} \unrhd x_{2} \unrhd x_{3} \unrhd x_{1}$ \\
4 & 0.3407 & 0.3904 & 0.3507 & 0.4963 & 0.5444 & $x_{5} \unrhd x_{4} \unrhd x_{2} \unrhd x_{3} \unrhd x_{1}$ \\
6 & 0.3475 & 0.3978 & 0.3505 & 0.4993 & 0.5436 & $x_{5} \unrhd x_{4} \unrhd x_{2} \unrhd x_{3} \unrhd x_{1}$ \\
8 & 0.3511 & 0.4027 & 0.3497 & 0.5006 & 0.5433 & $x_{5} \unrhd x_{4} \unrhd x_{2} \unrhd x_{1} \unrhd x_{3}$ \\
10 & 0.3533 & 0.4061 & 0.3490 & 0.5010 & 0.5430 & $x_{5} \unrhd x_{4} \unrhd x_{2} \unrhd x_{1} \unrhd x_{3}$ \\
\hline
\end{tabular}


Table 11. Relative closeness coefficients obtained by IP-GIVPFOWAD.

\begin{tabular}{ccccccc}
\hline Value of $\boldsymbol{p}$ & $\boldsymbol{r}\left(\boldsymbol{x}_{\mathbf{1}}\right)$ & $\boldsymbol{r}\left(\boldsymbol{x}_{\boldsymbol{2}}\right)$ & $\boldsymbol{r}\left(\boldsymbol{x}_{\boldsymbol{3}}\right)$ & $\boldsymbol{r}\left(\boldsymbol{x}_{\mathbf{4}}\right)$ & $\boldsymbol{r}\left(\boldsymbol{x}_{\mathbf{5}}\right)$ & Ranking order \\
\hline 1 & 0.3194 & 0.3653 & 0.3383 & 0.4602 & 0.5419 & $x_{5} \unrhd x_{4} \unrhd x_{2} \unrhd x_{3} \unrhd x_{1}$ \\
2 & 0.3256 & 0.3894 & 0.3333 & 0.4806 & 0.5472 & $x_{5} \unrhd x_{4} \unrhd x_{2} \unrhd x_{3} \unrhd x_{1}$ \\
4 & 0.3374 & 0.4030 & 0.3344 & 0.4921 & 0.5450 & $x_{5} \unrhd x_{4} \unrhd x_{2} \unrhd x_{1} \unrhd x_{3}$ \\
6 & 0.3437 & 0.4096 & 0.3350 & 0.4970 & 0.5428 & $x_{5} \unrhd x_{4} \unrhd x_{2} \unrhd x_{1} \unrhd x_{3}$ \\
8 & 0.3472 & 0.4139 & 0.3349 & 0.4993 & 0.5414 & $x_{5} \unrhd x_{4} \unrhd x_{2} \unrhd x_{1} \unrhd x_{3}$ \\
10 & 0.3495 & 0.4169 & 0.3345 & 0.5002 & 0.5406 & $x_{5} \unrhd x_{4} \unrhd x_{2} \unrhd x_{1} \unrhd x_{3}$ \\
\hline
\end{tabular}

Table 12. Distances of $x_{1}$ and $x_{2}$ from $x^{+}$.

\begin{tabular}{cccccc}
\hline$D\left(x_{1}, x^{+}\right)$ & 0.5800 & 0.2550 & 0.3000 & 0.2800 & 0.3250 \\
\hline$D\left(x_{2}, x^{+}\right)$ & 0.5800 & 0.2550 & 0.3000 & 0.2800 & 0.3250 \\
\hline
\end{tabular}

- Also:

$$
\begin{aligned}
& P-G I V P F O W A D\left(x_{1}, x^{+}\right) \\
& \quad=P-G I V P F O W A D\left(x_{2}, x^{+}\right)=0.3561,
\end{aligned}
$$

and:

$$
\begin{aligned}
I P- & \operatorname{GIVPFOW} A D\left(x_{1}, x^{+}\right) \\
& =I P-G I V P F O W A D\left(x_{2}, x^{+}\right)=0.33340,
\end{aligned}
$$

indicate that $x_{1}$ and $x_{2}$ are of the same preference. Hence, in such cases, Liu et al.'s [21] distance measure (Eq. (8)) is not proper choice for application.

- Scoring of alternatives $x_{1}$ and $x_{2}$ with respect to criteria $C_{3}$ and $C_{4}$ by using Peng and Yang's [7] score and accuracy functions (Eqs. (4) and (5)) is as follows: $s\left(C_{3}\left(x_{1}\right)\right)=s\left(C_{3}\left(x_{2}\right)\right)=-0.0250$. However, it should be noted that $C_{3}\left(x_{1}\right)$ and $C_{3}\left(x_{2}\right)$ are represented by different IVPFNs. Also, while $s\left(C_{5}\left(x_{1}\right)\right)=s\left(C_{5}\left(x_{2}\right)\right)=0.0250, C_{5}\left(x_{1}\right)$ and $C_{5}\left(x_{2}\right)$ are represented by different IVPFNs;

- Regarding accuracies, we have $a\left(C_{3}\left(x_{1}\right)\right)=$ $s\left(C_{5}\left(x_{1}\right)\right)=0.4250$ and $a\left(C_{3}\left(x_{2}\right)\right)=s\left(C_{5}\left(x_{2}\right)\right)=$ 0.5550 , but $\left.C_{3}\left(x_{1}\right)\right) \neq C_{5}\left(x_{1}\right)$ and $\left.C_{3}\left(x_{2}\right)\right) \neq$ $C_{5}\left(x_{2}\right)$. Hence, we can not proceed to make decisions in the right direction through the Liu et al. [21] approach;

- Representing the performance of alternatives $x_{3}$ and $x_{4}$ by IVPFNs $C_{3}\left(x_{4}\right)=([0.1,0.2],[0.04,0.37])$ and $C_{3}\left(X_{5}\right)=[(0.2,0.3],[0.05,0.6])$, respectively, in terms of the criterion $C_{4}$ in the decision matrix as shown in Table 4 are by using Peng and Yang's [7] score and accuracy functions (Eqs. (4) and (5)), we have $s\left(C_{3}\left(x_{4}\right)\right)=-01400 ; s\left(C_{3}\left(x_{5}\right)\right)=-0.1400$ and $a\left(C_{3}\left(x_{4}\right)\right)=0.2700 ; a\left(C_{3}\left(x_{5}\right)\right)=0.2700$. This result shows that $C_{3}\left(x_{4}\right)$ and $C_{3}\left(x_{5}\right)$ are equivalent while, in fact, they are different;
- The above statement shows that by the Peng and Yang's [7] score and accuracy functions, we cannot determine the scoring and accuracy of $C_{3}\left(x_{4}\right)$ and $C_{3}\left(x_{5}\right)$, and we may fail to find the positive or negative ideal solution. Hence, we cannot proceed to make decisions by Liu et al. [21] approach.

\section{Conclusions and scope of future work}

In order to overcome the existing shortcomings in the literature, we successfully incorporated four important parameters of membership, non-membership, strength, and direction of commitment and introduced a new accuracy function for interval valued Pythagorean Fuzzy Sets (PFSs). Furthermore, a new IVPF $p$ distance measure for interval-valued Pythagorean fuzzy numbers was proposed and used along with the existing weighted averaging operators to deal with an example of Multi-Criteria Decision Making (MCDM) problem. In any field of model evaluation and assessment of the quality of prediction, the estimator score and accuracy functions may be utilized in the future research. Also, the proposed distance measure may be used in multilabel ranking metrics, regression metrics, and clustering metrics.

\section{References}

1. Zadeh, L.A. "Fuzzy sets", Information and Control, 8(3), pp. 338-353 (1965).

2. Atanassov, K.T. "Intuitionistic fuzzy sets", Fuzzy Sets and Systems, 20(1), pp. 87-96 (1986).

3. Garg, H., Agarwal, N., Tripathi, A. "Some improved interactive aggregation operators under interval-valued intuitionistic fuzzy environment and its application to decision making process", Scientia Iranica, 24(5), pp. 2581-2604 (2017).

4. Yager, R.R. "Pythagorean membership grades in multicriteria decision making", IEEE Transaction on Fuzzy Systems, 22, pp. 958-965 (2014).

5. Yager, R.R. and Abbasov, A.M. "Pythagorean membership grades, complex numbers and decision making", International Journal of Intelligent Systems, 28, pp. $436-452$ (2013). 
6. Zhang, X.L. and Xu, Z.S. "Extension of TOPSIS tomulti-criteria decision making with Pythagorean fuzzy sets", International Journal of Intelligent Systems, 29, pp. 1061-1078 (2014).

7. Peng, X.D. and Yang, Y. "Fundamental properties of interval-valued Pythagorean fuzzy aggregation operators", International Journal of Intelligent Systems, $\mathbf{3 0}$, pp. 1-44 (2015).

8. Peng, X.D. "New operations for interval-valued Pythagorean fuzzy set", Scientia Iranica, 26(2), pp. 1049-1076 (2019). DOI: 10.24200/sci.2018.5142.1119

9. Zhang, X.L. "Multicriteria Pythagorean fuzzy decision analysis: a hierarchical QUALIFLEX approach with the closeness index-based ranking methods", Information Sciences, 330, pp. 104-124 (2016).

10. Chen, T.Y. "Multiple criteria decision analysis under complex uncertainty: A pearson-like correlation -based Pythagorean fuzzy compromise approach", International Journal of Intelligent Systems, pp. 1-38 (2018). https://doi.org/10.1002/int.22045

11. Tang, X. and Wei, G. "Some generalized Pythagorean 2-tuple linguistic Bonferroni mean operators in multiple attribute decision making", Journal of Algorithms and Computational Technology, 12(4), pp. 387-398 (2018). https://doi.org/10.1177/1748301818791506

12. Peng, X.D. and Selvachandran, G. "Pythagorean fuzzy set: state of the art and future directions", Artificial Intelligence Review, 52(3), pp. 1873-1927 (2019). DOI: $10.1007 / \mathrm{s} 10462-017-9596-9$

13. Peng, X.D. and Yang, Y. "Pythagorean fuzzy choquet integral based MABAC method for multiple attribute group decision making", International Journal of Intelligent Systems, 31(10), pp. 989-1020 (2016).

14. Peng, X.D., Yuan, H.Y., and Yang, Y. "Pythagorean fuzzy information measures and their applications", International Journal of Intelligent Systems, 32(10), pp. 991-1029 (2017).

15. Grzegorzewski, P. "Distance between intuitionistic fuzzy sets and/or interval-valued fuzzy sets based on the Hausdorff metric", Fuzzy Sets and Systems, 148, pp. 319-328 (2004).

16. Szmidt, E. and Kacprzyk, J. "Distance between intuitionistic fuzzy sets", Fuzzy Sets and Systems, 114, pp. 505-518 (2000).

17. Xu, Z.S. and Chen, J. "An overview of distance and similarity measures of intuitionistic fuzzy sets", International Journal of Uncertainty, Fuzziness and Knowledge-Based Systems, 16, pp. 529-555 (2008).

18. Dubois, D. and Prade, H., Fuzzy Sets and Systems: Theory and Applications, New York: Academic Press (1980).

19. Zeng, W.Y. and Guo, P. "Normalized distance, similarity measure, inclusion measure and entropy of intervalvalued fuzzy sets and their relationships", Information
Sciences, 178, pp. 1334-1342 (2008).

20. Deqing, L. and Wenyi, Z. "Distance measure of Pythagorean fuzzy sets", International Journal of Intelligent Systems, 33, pp. 348-361 (2018).

21. Liu, Y., Qin, Y., and Han, Y. "Multiple criteria decision making with probabilities in interval-valued Pythagorean fuzzy setting", International Journal of Fuzzy Systems, 20(2), pp. 558-571 (2018).

22. Peng, X.D. and Yang, Y. "Some results for Pythagorean fuzzy sets", International Journal of Intelligent Systems, 30, pp. 1133-1160 (2015).

23. Garg, H. "A novel improved accuracy function for interval valued Pythagorean fuzzy sets and its applications in the decision-making process", International Journal of Intelligent Systems, 32(12), pp. 1247-1260 (2017).

24. Yager, R.R., Engemann, K.J., and Filev, D.P. "On the concept of immediate probabilities", International Journal of Intelligent Systems, 10, pp. 373-397 (1995).

25. Wei, G.W. and Merigo, J.M. "Methods for strategic decision-making problems with immediate probabilities in intuitionistic fuzzy setting", Scientia Iranica, 19(6), pp. 1936-1946 (2012).

\section{Biographies}

Tanuj Kumar, PhD, is currently working as an Assistant Professor in the Department of Mathematics, SRM Institute of Science and Technology, Delhi NCR Campus, Ghaziabad, India. He received his MSc degree from Choudhary Charan Singh University, Meerut, India, in 2006 and MTech in Applied \& Computational Mathematics from Jaypee Institute of Information Technology, Noida, India, in 2011. Also, he received $\mathrm{PhD}$ degree in Mathematics from Jaypee University of Information Technology, Waknaghat, Solan, India, in 2015. His areas of research interest are fuzzy Information measures, decision making, and pattern recognition and applications.

Rakesh Kumar Bajaj, $\mathrm{PhD}$, received his BSc degree with honors in Mathematics from Banaras Hindu University, Varanasi, and MSc degree from the Indian Institute of Technology, Kanpur, in 2000 and 2002, respectively. Also, he received his $\mathrm{PhD}$ in Mathematics from Jaypee University of Information Technology (JUIT), Waknaghat, in 2009. He is now an Associate Professor and has been working in the Department of Mathematics at Jaypee University of Information Technology, since 2003. His research interests include fuzzy information measures, pattern recognition, fuzzy clustering, fuzzy statistics, and fuzzy mathematics in image processing.

Mohd Dilshad Ansari, PhD, is currently working as an Assistant Professor in the Department of Computer 
Science \& Engineering at CMR College of Engineering \& Technology, Hyderabad, India. He received his MTech in Computer Science and Engineering in 2011 and his $\mathrm{PhD}$ from Jaypee University of Information Technology, Waknaghat, Solan, HP, India, in 2018. He has published more than 25 papers in international journals and conferences. He is a member of various technical/professional societies such as IEEE, UACEE, and IACSIT. Also, he is currently a guest editor and editorial/reviewer board member of some Scopusindex journals and a technical programme committee member of various reputed journals/conferences. His research interest includes digital and fuzzy image processing, IoT, and cloud computing. 